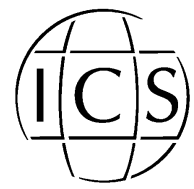

www.ics-elsevier.com

\title{
Killing kinetics of group B streptococci for penicillin in combination with gentamicin
}

\author{
P. Melin *, S. Lorquet, M.P. Hayette, P. De Mol \\ Belgian Reference Laboratory for Group B Streptococci, University Hospital of Liege, 4000 Liege, Belgium
}

\begin{abstract}
In vitro synergism and killing kinetics studies of penicillin in combination with gentamicin were performed with group B streptococci recently isolated in Belgium. The expected accelerated killing was not observed when compared with penicillin alone. (C) 2005 Elsevier B.V. All rights reserved.
\end{abstract}

Keywords: Group B streptococcus; Antimicrobial susceptibility; Antimicrobial synergy

\section{Introduction}

Associated with high morbidity and mortality, severe group B streptococcal (GBS) infections should be treated promptly with antimicrobial agents characterized by both a good diffusion at the site of infection and a short bactericidal lag time. GBS are uniformly susceptible to penicillin or ampicillin at concentrations usually achieved in blood or cerebrospinal fluid. Gentamicin alone is not regarded as clinically useful in the treatment of GBS infections; however, to accelerate GBS killing, penicillin or another $\beta$-lactam given in combination with gentamicin is recommended to start the therapy [1,2]. Minimum inhibitory concentrations (MIC) of gentamicin (G) for GBS isolated in Belgium range from 16 to 256 $\mathrm{mg} / \mathrm{L}$ [3]. These observed MICs of gentamicin are often higher than G-MICs for Enterococcus faecalis with low-level resistance, but lower than the G-MICs of E. faecalis with a high-level of resistance to G. This study was undertaken to determine conditions required for eradication of GBS in vitro, especially for strains with the highest MICs determined for gentamicin. The potential synergism and killing kinetics of penicillin and gentamicin individually or in combination (ratio 1:1), at different concentrations, were investigated against strains of GBS.

\footnotetext{
* Corresponding author. Tel.: +32 436624 39; fax: +32 43662440 .

E-mail address: Pierrette.Melin@chu.ulg.ac.be (P. Melin).
} 


\section{Material and methods}

\subsection{Bacterial isolates}

Six strains of GBS isolated from clinically important sources from neonates or adults with invasive infections, submitted to the Belgian reference laboratory for GBS in 20022003 and preserved at $-70{ }^{\circ} \mathrm{C}$, were selected either for their known low MIC or higher MIC to gentamicin (16 to $128 \mathrm{mg} / \mathrm{L}$ ). As positive and negative controls, for comparison in the synergy and timed antibiotic-killing studies, two strains of E. faecalis either with low or high level of resistance to gentamicin were used.

\subsection{Determination of MICs and kinetic studies: determination of killing curves}

Determinations of MICs of penicillin and gentamicin were performed for GBS and $E$. faecalis isolates using Etest strips according to the Etest ABBiodisk method (Sweden), respectively on Muller Hinton agar supplemented or not with 5\% of sheep blood.

Timed killing assays with penicillin were performed according to an Etest ABBiodisk original procedure. Each isolate was tested twice. A killing curve master plate was prepared by flooding a 14-cm plate of Muller Hinton agar (with or without sheep blood agar) with a 1:10 dilution of a $0.5 \mathrm{McF}$ arland inoculum suspension. The excess fluid was pipetted and drained and the plate was dried for $15 \mathrm{~min}$ in an incubator. Then six Etest strips of penicillin were applied upon the surface using a template. A growth control area $\left(3 \times 3 \mathrm{~mm}^{2}\right)$ was sampled with a $1-\mu 1$ loop and then streaked in the "control" quadrant of CFU (colony forming units) of a sheep blood agar plate, the " $t=0$ plate". Along an Etest strip at the levels of the known $\mathrm{MIC} \times 4, \mathrm{MIC} \times 8$ and $\mathrm{MIC} \times 16$, areas were sampled and streaked in the respective CFU quadrants of the same $t=0$ plate. The master plate was then re-incubated and the CFU $t=0$ plate was incubated at $35^{\circ} \mathrm{C}$. After $2 \mathrm{~h}$, the master plate was taken out of the incubator and sampled as previously along another Etest strip: the control and MIC multiples. The samples were streaked in the quadrants of another blood agar plate, the $t=2$ plate. Again the master plate was re-incubated and the $t=2 \mathrm{CFU}$ plate incubated. The procedure was repeated at incubation intervals $4 \mathrm{~h}, 8 \mathrm{~h}$, and $20 \mathrm{~h}$. After overnight incubation, the colonies or the surviving organisms per quadrant were enumerated on the initial $t=0$ plate and repeatedly on the plates prepared after $2,4,8$ and $20 \mathrm{~h}$. The amounts of CFU/9 $\mathrm{mm}^{2}$ were plotted versus sampling time for the control and MIC multiples.

\subsection{Synergy experiments}

Determination of MICs in a combination test was performed according to an Etest ABBiodisk original procedure as follows. The Muller Hinton agar (supplemented or not with $5 \%$ sheep blood) plate was inoculated as for the determination of MICs of individual drugs, then a strip of gentamicin (range of $0.016-256 \mathrm{mg} / \mathrm{L}$ ) was placed on the agar surface, its position was marked and the plate was left for $1 \mathrm{~h}$ on the bench. After $1 \mathrm{~h}$, the strip was removed and a penicillin strip was positioned onto the imprint of the first antibiotic to have a ratio of $1: 1$, the gradients were then superimposed. Immediately the plate was transferred at $35{ }^{\circ} \mathrm{C}$ and incubated overnight. MICs of each antibiotic in the combination test was read and compared to the MICs of each antibiotic individually determined. A synergistic effect was considered to be present when MIC of drugs in the combination was $>2$ dilutions lower than the MIC of the most active single drug. 
A

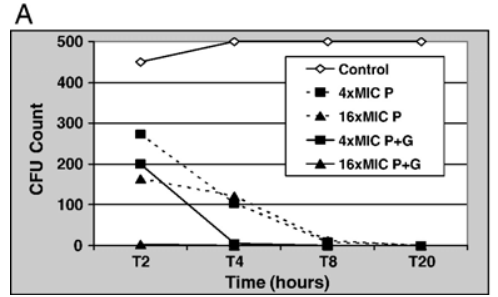

B

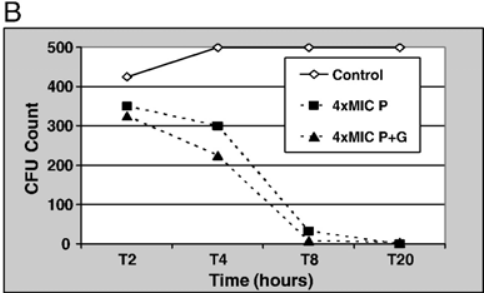

Fig. 1. Growth of E. faecalis without antibiotic (control) and median killing curves for gentamicin low level (A) or high level (B) resistance E. faecalis for penicillin $(\mathrm{P})$ alone or in combination with gentamicin $(\mathrm{P}+\mathrm{G})$ at different concentrations (4-fold and 16-fold penicillin MIC).

Indifference was reported when MIC of drugs in the combination was within \pm 1 dilution compared to single drugs.

Kinetic studies were performed according to a combination of the two ABBiodisk procedures described above. The kinetic testing procedure was performed after a previous step, in which gentamicin strips were left for $1 \mathrm{~h}$ upon the plate, further removed and replaced with penicillin strips. Timed killing curves determined for the combination at different concentrations were respectively compared to killing times observed with penicillin alone. The combination of antibiotics was considered synergistic to reduce the killing time when it eradicated GBS in a shorter time than penicillin alone.

\section{Results}

The range of MICs of penicillin for the 6 isolates of GBS was $0.032-0.047 \mathrm{mg} / \mathrm{L}$. For the E. faecalis, the MICs were respectively 1.5 and $6 \mathrm{mg} / \mathrm{L}$ for the isolates with a low level or a high level resistance to gentamicin.

Either for all GBS isolates or for the two E. faecalis strains, after overnight incubation, penicillin and gentamicin in combination were not more effective than penicillin alone to eradicate isolates in culture. MICs of penicillin in the combination were within +1 dilution compared to penicillin alone.

In the kinetic experiments, for all strains, growth of bacteria occurred in the absence of antibiotic (control) and rapidly exceeded $500 \mathrm{CFU}$ per sample. As expected, Fig. 1A shows the synergistic effect of penicillin plus gentamicin against the strain of E. faecalis with a low level of resistance to gentamicin. To eradicate the strain in culture submitted to
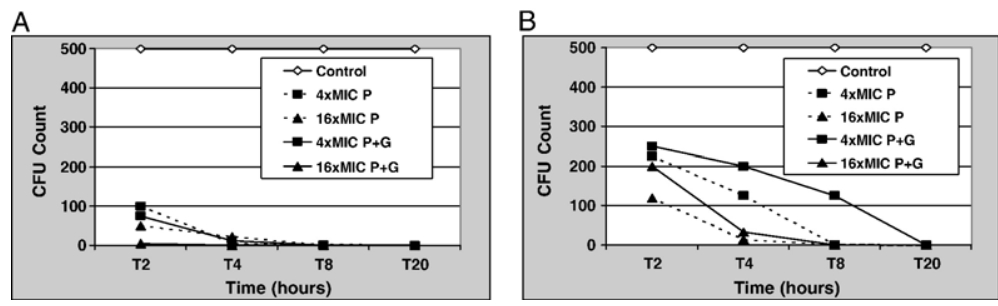

Fig. 2. Growth of GBS without antibiotic (control) and median killing curves for two strains of GBS for penicillin (P) alone or in combination with gentamicin $(P+G)$ at different concentrations (4-fold and 16-fold penicillin MIC). 
penicillin concentrations either of 4-fold the MIC or 16-fold, more than $8 \mathrm{~h}$ were necessary. When penicillin was associated to gentamicin, killing was enhanced: the killing times for concentrations of penicillin at 4-fold and 16-fold its MIC were, respectively, 4 and $2 \mathrm{~h}$. Fig. 1B illustrates the lack of synergism of penicillin plus gentamicin against the strain of E. faecalis highly resistant to gentamicin. The killing curves of penicillin alone or in combination with gentamicin are rather parallel and close to each other. In all the tested conditions, to eradicate the strain, overnight incubation was mandatory. On the contrary, compared with penicillin alone, nearly no accelerated killing was observed for any GBS isolate with the combination penicillin plus gentamicin even for a concentration of 16-fold MIC of penicillin (indifference); moreover, for 3 isolates, a reduced killing was observed in the combination tests (antagonism). Representative data are shown in the Fig. 2.

\section{Discussion}

For our kinetics studies and investigation for synergistic effect, performed according to original ABBiodisk procedures, the expected results observed with the strains of $E$. faecalis validated the procedures used in this study. But the observed results with GBS strains were quite different of expectation: no clear synergy was demonstrated with the combination penicillin plus gentamicin (1:1) used, independently of values of gentamicinMICs; moreover, the killing was reduced for half of the isolates of GBS by comparison with penicillin alone. These in vitro studies, the conditions of which vary considerably from the pharmacodynamic and pharmacologic conditions of clinical in vivo use of a combination therapy, just show that any $\beta$-lactam in combination with gentamicin did not demonstrate necessarily the expected accelerated killing of GBS. To determine which combination and ratio of antimicrobial agents could be used to achieve this goal, and to recommend wisely which regimen should be administered to treat patients with invasive GBS infections, further evaluation should be performed on these strains with other ratio or other $\beta$-lactams, in combination with gentamicin.

\section{References}

[1] CDC, Prevention of perinatal Group B streptococcal disease: Revised guidelines from CDC, MMWR 51 (RR11) (2002) 1-22.

[2] V. Schauf, et al., Antibiotic-killing kinetics of group B streptococci, J. Pediatr. 89 (1976) 194-198.

[3] P. Melin, Streptococcus agalactiae. In G. Ducoffre, Surveillance des maladies infectieuses par un réseau de laboratoires de microbiologie 2004. Tendances épidémiologiques 1983-2003, Institut Scientifique de la santé publique, section épidémiologie, Belgique, December 2005. 